\title{
Modified Leakage Rate Calculation Models of Natural Gas Pipelines
}

\author{
Qingmin Hou $\mathbb{D}^{1},{ }^{1}$ Daheng Yang, ${ }^{1,2}$ Xiaoyan Li, ${ }^{1}$ Guanghua Xiao, ${ }^{3}$ and Siu Chun Michael Ho ${ }^{4}$ \\ ${ }^{1}$ School of Energy and Building Engineering, Harbin University of Commerce, Harbin 150028, China \\ ${ }^{2}$ School of Food Engineering, Harbin University of Commerce, Harbin 150028, China \\ ${ }^{3}$ Department of Municipal and Environmental Engineering, Heilongjiang Institute of Construction Technology, \\ Harbin 150025, China \\ ${ }^{4}$ Department of Mechanical Engineering, University of Houston, Houston, TX 77004, USA
}

Correspondence should be addressed to Qingmin Hou; sspawn@163.com

Received 9 October 2020; Revised 11 November 2020; Accepted 24 November 2020; Published 7 December 2020

Academic Editor: Yufei Wang

Copyright (C) 2020 Qingmin Hou et al. This is an open access article distributed under the Creative Commons Attribution License, which permits unrestricted use, distribution, and reproduction in any medium, provided the original work is properly cited.

The leakage rate is an essential parameter for the risk assessment and failure analysis of natural gas pipelines. The leakage rate of a natural gas pipeline should be calculated quickly and accurately to minimize consequences. First, in this study, models to estimate the leakage rate of natural gas pipelines are reclassified, and the theoretical range of application for each model is also analysed. Second, the impact of the leakage on the flow rate upstream of the leak point is considered, and the method of successive approximation is used to realize this feedback effect of flow rate change. Then, a modified hole-pipe model is developed to calculate the natural gas leakage rate in this paper. Compared with the leakage rate calculated by the hole-pipe model, the leakage rate calculated by the modified hole-pipe model is smaller and closer to the actual leakage rate due to the consideration of the feedback effect of the flow rate change. Finally, the leakage rate curves of the hole-pipe model and the modified hole-pipe model under different $\mathrm{d} / \mathrm{D}$ conditions are obtained through simulation. The simulation results show that the modified hole-pipe model is able to calculate the leakage rate of any leak aperture, such as the hole-pipe model, and also at a higher accuracy level than the holepipe model.

\section{Introduction}

Natural gas is a high-quality, efficient, and clean source of energy. Since the 1970s, worldwide consumption of natural gas has accelerated, and research on natural gas exploitation, transportation, and storage has also increased to meet demands [1-10]. Pipeline infrastructure has been erected around the globe to support the natural gas operations. The sheer lengths of pipeline laid in a wide variety of environments inevitably lead to accelerated pipeline damage and failures in certain situations. International statistics of natural gas pipeline accidents show that artificial damage, construction errors, material defects, and corrosion are the common causes of natural gas pipeline leakages. Leakages can lead to fires and even deadly explosions; therefore, it is crucial to rapidly mitigate any pipeline accidents to reduce human, environmental, and corporate losses. Estimating the leakage rate of a damaged natural gas pipeline is the first step for predicting the area affected by the leakage and guiding any needed personnel evacuation.

Due to the importance of rapid leakage detection and analysis, many researchers have contributed to the development of leakage models. Montiel et al. first proposed the concept of the hole-pipe model and discussed the usage of small hole, large hole, and pipe models of pipeline leakage [11]. Zhou analysed the thermal process of slow discharge of natural gas storage tanks and established a corresponding mathematical model [12, 13]. Woodward and Mudan proposed an ideal fluid leakage model for small holes in compression vessels [14]. Based on their model, the Center for Chemical Process Safety (CCPS) proposed several approximate formulas for calculating leakage rates under certain conditions [15]. Young et al. proposed a simplified calculation model for the small hole leakage rate of high- 
pressure gas pipelines while considering the safety margin often required in practical situations, and due to the margin, the calculated results were larger than the actual results $[16,17]$. Arnaldos et al. performed a simple analysis of the leakage rate calculation for both the pipe model and the small hole model [18]. Levenspiel developed a leakage model for when the pipeline is completely fractured and analysed the pressure drop along the pipeline. However, the model was developed based on the assumption that the pressure at the starting point remains constant and the gas flow and leakage in the pipe are adiabatic processes [19]. Dong calculated the steady state gas leakage of the long-distance pipeline [20]. Yang et al. established a steady state leakage model of a nonisothermal long-distance pipeline [21].

From these studies, the following conclusions can be drawn: The leakage models are usually classified into three types: (1) a small hole model which can be used to calculate the leakage rate of small holes. This model not only ignores the friction along the pipelines but also does not consider the effect of leakage on the pressure in the pipelines. Therefore, the error of this model is large, especially when the leak point is not small. (2) A pipe model to calculate the leakage rate of a natural gas pipeline when the pipeline is completely broken. This model assumes isentropic release, and a constant pressure is assumed at an initial point in the pipe. The pressure drop along the pipe is taken into account. This model provides accurate predictions for the case where the natural gas pipeline is completely broken, but it cannot be applied to the flow through holes with a diameter smaller than the pipe diameter. (3) The hole-pipe model, which was first proposed by Montiel et al., then became the critical cornerstone of the field. Theoretically, this widely used model can calculate the leakage rate for various apertures. The friction along the pipelines and the effect of leakage on the pressure in the pipelines are both taken into account in this model. However, the effect of leakage on the flow rate of natural gas pipelines is not considered. When the hole diameter is larger than the typical small hole diameter and smaller than the pipeline diameter, the error still cannot be ignored.

Based on the above analysis, this paper reclassifies the original leakage models. Furthermore, four new models for calculating the leakage rate are developed: the storage tank model, small hole model, modified hole-pipe model, and pipe model. The characteristics of each model and the specific conditions of their application are detailed. Considering how flow rate changes in natural gas pipelines can cause a feedback phenomenon, a modified hole-pipe model is proposed in this paper for calculating the leakage rate of various apertures, and an example is used to verify the superiority and rationality of the models.

\section{Reclassification of the Leakage Rate Calculation Models}

In the traditional classification, leakage rate calculation models are usually divided into three categories. According to the actual situations, this paper reclassifies them into four categories and gives the applicable scope of each model.
The system analysed is shown schematically in Figure 1 [11]. As shown in this figure, there is a length of pipeline $L_{e}$ after which there is a hole with a certain diameter through which the pressure release takes place.

Locations of interest include point 1 , at the beginning of the pipe

Point 2, at the center of the pipeline, on the same vertical axis as the leak point

Point 3, at the leak point

Point 4, at the outside of the pipe, exposed to atmospheric pressure

To calculate the leakage rate, the following hypotheses are assumed: (a) a model of essentially one-dimensional flow; (b) isentropic flow at the release point and adiabatic flow in the pipe; and (c) the gas behaves as an ideal gas. An air compression factor is added to the ideal gas equation of state to reduce the difference from the actual gas.

By applying the energy and momentum equations to the adiabatic flow through a pipeline, the following equation is obtained [22]:

$$
\frac{k+1}{k} \ln \left(\frac{P_{1} T_{2}}{P_{2} T_{1}}\right)+\frac{M}{R G^{2}}\left(\frac{P_{2}^{2}}{T_{2}}-\frac{P_{1}^{2}}{T_{1}}\right)+\left(\frac{4 \lambda X_{e}}{D}\right)=0 .
$$

In this expression, $\lambda$ is the Fanning friction factor. The natural gas leakage rate $K$ at the hole can be calculated using the following expression, which is obtained from the continuity equation and the law of ideal gases for an isentropic expansion:

$$
K=C_{D} A_{\text {or }} P_{2} \sqrt{\frac{M}{Z R T_{2}} \frac{2 k}{k-1}\left[\left(\frac{P_{a}}{P_{2}}\right)^{2 / k}-\left(\frac{P_{a}}{P_{2}}\right)^{k+1 / k}\right]},
$$

where $C_{D}$ is the flow correction coefficient of the leaking hole [23] and is usually set to a value between 0.6 and 1.0. This coefficient is classified according to the shape of the hole. When the exact shape of the hole is unknown, a conservative value of 1.0 is usually recommended. This value was used in this paper [11].

The flow rate at the leak point depends on whether the flow is sonic or subsonic, which can be determined by the critical pressure ratio $(\mathrm{CPR})$ :

$$
\mathrm{CPR}=\frac{P_{a}}{P_{2 c}}=\left(\frac{2}{k+1}\right)^{k / k-1},
$$

where $P_{2 c}$ is the critical pressure at point 2. If the pressure $P_{2}$ at point 2 increases gradually, the speed of gas leakage will increase until it is equal to the local sound speed. At this moment, if $P_{2}$ continues to increase, then the gas leakage rate remains constant and is always equal to the local sound speed and demarcates a critical stage of flow.

When $P_{a} / P_{2}<\mathrm{CPR}$, then critical flow leakage has been reached, and equation (4) is substituted into equation (2) for the expression of the leakage: 


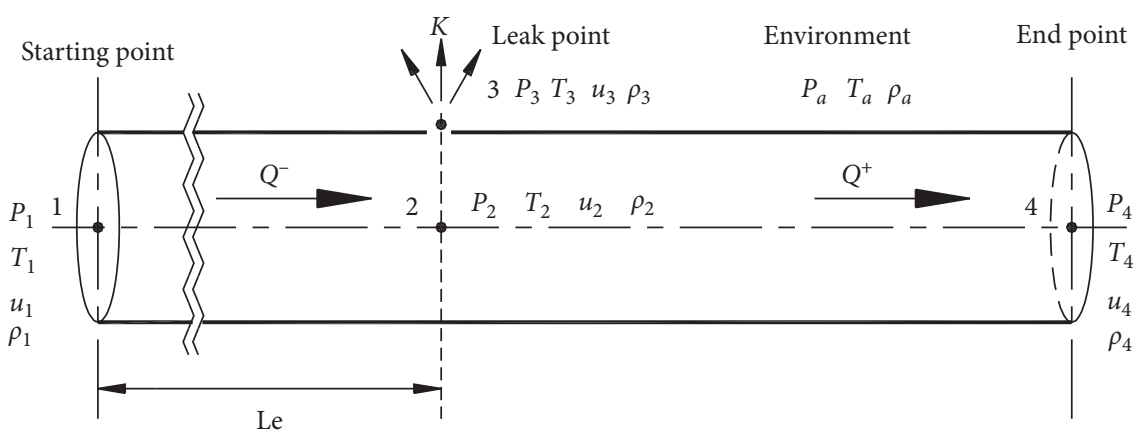

Figure 1: Schematic diagram of gas pipe leakage.

$$
K=C_{D} A_{\mathrm{or}} P_{2} \sqrt{\frac{M k}{\mathrm{ZRT}_{2}}\left(\frac{2}{k+1}\right)^{k+1 / k-1}} .
$$

When $P_{a} / P_{2} \geq \mathrm{CPR}$, the leakage is at subcritical flow, and the leakage can be calculated by equation (2). Equations (2) and (4) are general formulas for calculating the natural gas leakage rate.

According to different but approximate conditions, natural gas pipeline leakage models (i.e., storage tank model, small hole model, modified hole-pipe model, and pipe model) are established in this paper. These four models will be detailed in the upcoming section.

2.1. Storage Tank Model. When a gas storage tank leaks, the following approximate assumptions can be made because of the large size of the tank: (a) the pressure inside the storage tank is not affected by the leakage, and the parameter values at point 2 remain unchanged both before and after the leakage. (b) The pressure loss caused by the friction between the container and the gas flow after the leakage is ignored. By these two assumptions, the parameters of point 1 are used to replace the parameter value of point 2 , and the leakage rate can be calculated by formulas (2) and (4), which comprise the storage tank model. This model has been widely used in the accurate calculation of the leakage of large containers, such as storage tanks [18].

All these aspects make this model adequate for the prediction of release through a hole in a tank but not for leakages in natural gas pipelines. Because the gas pipeline is different from the storage tank, especially when point 2 is far from point 1 , the pressure loss and velocity caused by internal friction must be considered. The related parameters of point 2 are not exactly the same as those at point 1. The leakage rate calculated by this model is larger than the actual leakage rate. Therefore, in general, the storage tank model is not suitable for the calculation of the natural gas pipeline leakage rates. Only when point 2 is close to point 1 and the hole diameter is very small can the storage tank model be used to calculate the leakage rate for natural gas pipelines.

2.2. Small Hole Model. A small hole model is used to calculate the natural gas pipeline leakage rate when the hole is small. This model takes into account the effect of friction in the natural gas pipeline. Because the leakage is small, the effect of leakage on the pressure and flow rate in the natural gas pipeline is neglected. Assuming that the coefficient of friction is the same along the whole pipeline, the relationship between point 1 and point 2 can be defined by the following equations [11].

$$
\frac{k+1}{2} \ln \left[\frac{M a_{2}^{2}}{M a_{1}^{2}} \frac{2+(k-1) M a_{1}^{2}}{2+(k-1) M a_{2}^{2}}\right]-\left(\frac{1}{M a_{1}^{2}}-\frac{1}{M a_{2}^{2}}\right)+\frac{4 \lambda X_{\varepsilon}}{D}=0
$$

$$
\begin{aligned}
& \frac{T_{2}}{T_{1}}=\frac{Y_{1}}{Y_{2}}, \\
& \frac{P_{2}}{P_{1}}=\frac{M a_{1}}{M a_{2}} \sqrt{\frac{Y_{1}}{Y_{2}}}, \\
& \frac{\rho_{2}}{\rho_{1}}=\frac{M a_{1}}{M a_{2}} \sqrt{\frac{Y_{2}}{Y_{1}}},
\end{aligned}
$$

where

$$
Y_{i}=1+\left(\frac{k-1}{2}\right) \cdot \mathrm{Ma}_{i}^{2}
$$

In these expressions, $\mathrm{Ma}$ is the Mach number, which can be calculated as follows:

$$
\mathrm{Ma}_{i}=\frac{u_{i}}{c}=\frac{u_{i}}{\sqrt{\mathrm{kZRT} / M}}
$$

Under these conditions, the following relationships apply:

When $P_{a} / P_{2}<\mathrm{CPR}$, there is a critical flow at the leak point, and the leakage rate can be calculated by equation (4).

When $P_{a} / P_{2} \geq \mathrm{CPR}$, there is a subcritical flow at the leak point, and the leakage rate can be calculated by equation (2).

The small hole model considers the pressure loss caused by the friction of the natural gas pipeline and is more accurate than the storage tank model. However, because the small hole model neglects the effect of leakage on the pressure and flow rate in a natural gas pipeline, the parameters at point 2 remain unchanged upstream and downstream of the leakage. This hypothesis is still different 
from the actual situation. Therefore, only when the leakage hole is very small can the small hole model be used to calculate the leakage rate.

2.3. Pipe Model. The pipe model is used for the case of the complete rupture of the natural gas pipeline or when the leakage diameter is close to the pipe diameter. The state of the natural gas in the pipe is the same as if the gas is in atmospheric conditions. According to the conservation of flow rate, the leakage rate is equal to the flow rate in a natural gas pipeline. Thus, the formula for calculating the natural gas leakage rate is as follows [18]:

$$
K=Q=C_{D} A_{\text {or }} \sqrt{\frac{2 M}{Z R} \frac{k}{k-1} \frac{T_{2}-T_{1}}{\left(T_{1} / P_{1}\right)^{2}-\left(T_{2} / P_{2}\right)^{2}}}
$$

At this time, because the pipeline is completely broken, $P_{2}=P_{3}=P_{a}$, and $T_{2}$ can be obtained by using equation (6). The model gives good predictions for the case of the complete destruction of the natural gas pipelines, but it cannot be applied to the flow through holes with a diameter smaller than the natural gas pipeline diameter.

2.4. Modified Hole-Pipe Model. Before introducing the modified hole-pipe model, the hole-pipe model should be introduced first. Two aspects have been taken into account by the hole-pipe model: the pressure loss caused by the friction of the pipeline and the effect of leakage on the pressure in the pipeline. However, the effect of the leakage on the flow rate upstream of the leak point is not considered.

In the hole-pipe model, the leakage rate is also calculated by equations (2) and (4). Equations (5)-(10) express the relationship of parameters between points 1 and 2, and these equations are substituted into equations (2) and (4) to reflect the consideration of pressure loss caused by the friction of the natural gas pipeline.

For the critical flow at the leak point hole, the relationship of parameters between points 2 and 3 is defined as follows:

$$
\left\{\begin{array}{l}
P_{3}=\left[\frac{2}{(k+1)}\right]^{k /(k-1)} P_{2}, \\
T_{3}=\left[\frac{2}{(k+1)}\right] T_{2}, \\
\rho_{3}=\left[\frac{2}{(k+1)}\right]^{1 /(k-1)} \rho_{2} .
\end{array}\right.
$$

For the subcritical flow at the leak point hole, the pressures at point 3 and the environment are same, so the relationship of parameters between points 2 and 3 is defined as follows:

$$
\left\{\begin{array}{l}
P_{3}=P_{a}, \\
T_{3}=\left(\frac{P_{a}}{P_{2}}\right)^{(k-1) / k} T_{2}, \\
\rho_{3}=\left(\frac{P_{a}}{P_{2}}\right)^{1 / k} \rho_{2} .
\end{array}\right.
$$

Equations (12) and (13) are used to calculate $P_{2}$ after leakage occurs, and this can reflect the effect of leakage on the pressure, but the calculations are complicated.

The above is the main introduction of the hole-pipe model.

The modified hole-pipe model proposed in this paper is also used to calculate the gas leakage rate when the leak point hole diameter is larger than that of the small hole model. Because the hole diameter and leakage rate are larger, the influence of the following two aspects are considered:

(a) The effect of leakage on the pressure in the natural gas pipeline. The pressure at leak point $P_{2}$ will be reduced after leakage occurs, and the corresponding leakage rate will change. This effect has been taken into account by the hole-pipe model, but it requires complicated calculations. This effect is also considered in the modified hole-pipe model, and the successive approximation method is used to avoid complicated calculations.

(b) The effect of leakage on the flow rate upstream of the leak point. According to the law of conservation of mass, the flow rate upstream of the leak point should be equal to the sum of the leakage rate and the flow rate downstream the leak point. Thus, the flow rate upstream of the leak point will change, leading to a change in the pressure in the pipeline. The change in pressure further affects the leakage rate, which in turn affects the flow rate upstream of the leak point. This series of events acts as a feedback loop. The effect of leakage on the flow rate upstream of the leak point will extend to the vicinity of the source, and this is not taken into account by the hole-pipe model but is considered in the proposed modified hole-pipe model.

For convenience of calculation, the pressure $P_{4}$ at the end of the pipe is assumed to be a fixed value. In this model, the method of successive approximation is used to calculate the natural gas leakage rate. The concrete steps are as follows:

(1) The flow rate upstream of the leak point $Q^{-}$is known, and the leakage rate $K$ and the pressure of point $2 P_{2}$ are calculated by the small hole model

(2) If $P_{2}>P_{4}$, then the pipe flow rate after the leak point $Q^{+}$can be obtained according to pressures $P_{2}$ and $P_{4}$. According to mass conservation, the natural gas pipeline flow rate before the leak point can be obtained by $Q^{-}=K+Q^{+}$, and $Q^{-}$is inserted into step 1 to calculate the new $K^{\prime}$ and $P_{2}$ '. This process is 
iterated until the calculation results of the natural gas leakage rate tend to be stable.

(3) If $P_{2} \leq P_{4}$, then the natural gas pipeline flow rate after the leak point $Q^{+}$is 0 . According to the mass conservation, $Q^{-^{\prime}}=K$ can be obtained, and $Q^{-'}$ is inserted into step 1 to calculate the new $K^{\prime}$ and $P_{2}$ '. This process is continued until the calculation results of the natural gas leakage rate are stable.

The flowchart of the modified hole-pipe model is shown in Figure 2, where $\sigma$ represents the threshold.

Through the above specific steps, it can be found that the $P_{2}$ ' and $P_{2}$ obtained after a cycle will inevitably change. Thus, the influence of the change in $P_{2}$ ' on the flow state in the pipe should be considered. The flow at the leak point hole can then be classified as critical flow and subcritical flow, and the three states of flow in the pipeline and at the hole can be obtained:

(a) Subcritical flow in the pipeline and the critical flow at the leak point hole

$$
\left\{\begin{array}{l}
P_{2}^{\prime}>P_{1} M a_{1} \sqrt{\frac{2 Y_{1}}{k+1}}, \\
\frac{P_{a}}{P_{2}^{\prime}}<\mathrm{CPR} \Longleftrightarrow P_{2}^{\prime}>P_{2 c} .
\end{array}\right.
$$

At this point, the formula for calculating the leakage rate by the small hole model in step 1 is equation (4)

(b) Subcritical flow in the pipeline and the subcritical flow at the leak point

$$
\left\{\begin{array}{l}
P_{2}^{\prime}>P_{1} M a_{1} \sqrt{\frac{2 Y_{1}}{k+1}}, \\
\frac{P_{a}}{P_{2}^{\prime}} \geq \mathrm{CPR} \Longleftrightarrow P_{2}^{\prime} \leq P_{2 c} .
\end{array}\right.
$$

At this point, the formula for calculating the leakage rate by a small hole model in step 1 is equation (2)

(c) Critical flow in the natural gas pipeline and the critical flow at the leak point

$$
\left\{\begin{array}{l}
P_{2}^{\prime}<P_{1} M a_{1} \sqrt{\frac{2 Y_{1}}{k+1}}, \\
\frac{P_{a}}{P_{2}^{\prime}}<\mathrm{CPR} \Longleftrightarrow P_{2}^{\prime}>P_{2 c} .
\end{array}\right.
$$

At this point, the formula for calculating the leakage rate by a small hole model in step 1 is equation (4)

Similarly, the pressure $P_{2}$ ' at point 2 obtained in each cycle should be selected as described above. The modified

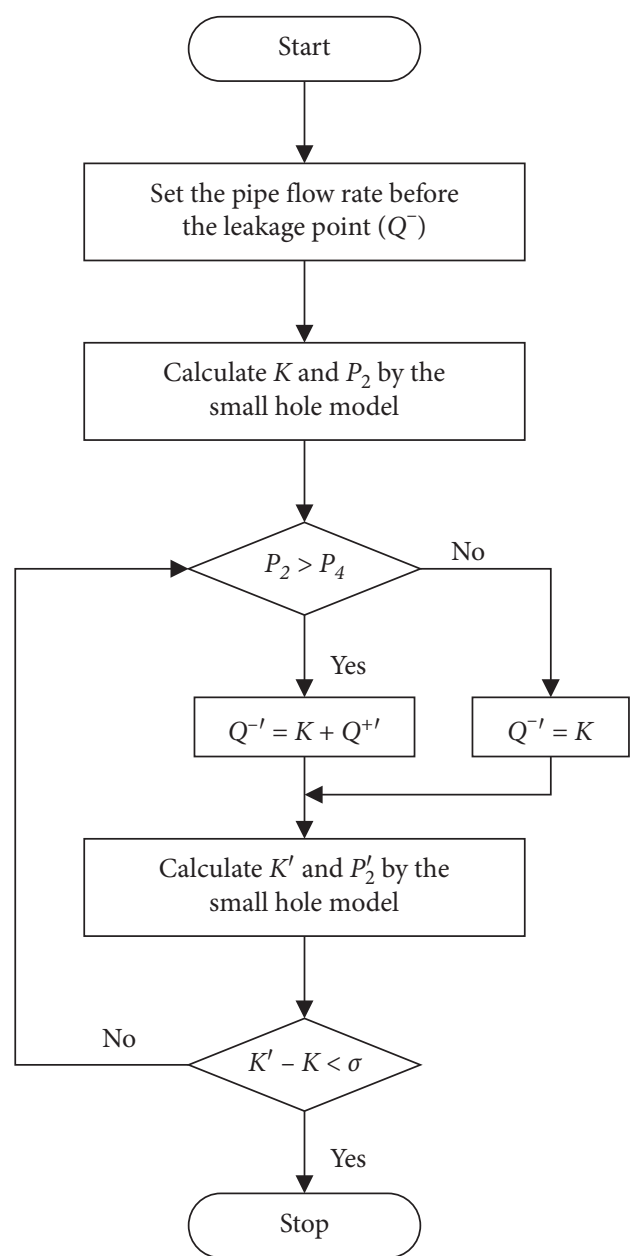

FIGURE 2: Flow chart of the modified hole-pipe model.

hole-pipe model takes into account the effect of leakage on the flow rate of the natural gas pipeline upstream and downstream the leak point, which is more in line with the actual situation. Moreover, the iterative approximation method used in this model can avoid the need for complicated mathematical derivations.

\section{Comparison between Models}

Due to the flammable and explosive character of natural gas, it is impractical to carry out leakage rate measurement experiments on real natural gas pipelines. Even if compressed air is used for the experiments, it is difficult to simulate several leakages of different aperture sizes to obtain different $d / D$ (the ratio of the leak point hole diameter to the pipeline diameter) values. Therefore, simulations are used in this paper to do a comparison between models.

3.1. Introduction of Calculation Example. To verify the effectiveness of the models, especially to compare the holepipe model and the modified hole-pipe model, this paper applies these models to the following accident scenarios. The parameters are shown in Table 1. 
TABLE 1: Basic computational parameters.

\begin{tabular}{lccc}
\hline$Q(\mathrm{~kg} / \mathrm{s})$ & $k$ & $R(\mathrm{~J} /(\mathrm{mol} \cdot \mathrm{K}))$ & $\operatorname{Re}$ \\
\hline 108 & 1.334 & 8.314 & 3000 \\
$D(\mathrm{~m})$ & $E(\mathrm{~mm})$ & $P_{a}(\mathrm{pa})$ & $M(\mathrm{~kg} / \mathrm{mol})$ \\
0.216 & 0.045 & $10^{5}$ & $16.48 \times 10^{-3}$ \\
$P_{1}(\mathrm{MPa})$ & $P_{4}(\mathrm{MPa})$ & $T_{1}(\mathrm{k})$ & $L e(\mathrm{~m})$ \\
18 & 6.8 & 293 & 1300 \\
\hline
\end{tabular}

3.2. Discussion and Comparison. Based on the above proposed models as well as the parameters, the relationship between the natural gas leakage rate and $\mathrm{d} / \mathrm{D}$ of different models under steady state conditions is shown in Figure 3. The natural gas leakage rates calculated by the storage tank model and the small hole model increase rapidly with increasing $d / D$. Only when the $d / D$ is small are the leakage rates of these two models close to the leakage rate of the modified hole-pipe model, which conforms to the scope of application of these two models. Only when the leakage point is very close to point 1 of the natural gas pipeline and the leakage hole is very small can the storage tank model be applied. It can also be seen from Figure 3 that the leakage rates of the modified hole-pipe model and the hole-pipe model show the same trend, both of which are very close. However, the leakage rate of the modified hole-pipe model is smaller than that of the hole-pipe model. The leakage rate difference at different $d / D$ values are shown in Figure 4. As $\mathrm{d} / \mathrm{D}$ increases, the difference between these two models first increases and then decreases.

When the leakage hole is very small, the small hole model can be applied. The diagram also shows that the natural gas leakage rate calculated by the storage tank model is larger than that of the small hole model, which is also consistent with the effect of the internal friction of the small hole model; therefore, the small hole model is more accurate for calculating the natural gas leakage rate than the storage tank model. Because leakage occurs, the pressure of point 2 will decrease (but will not decrease indefinitely), which makes the leakage rate calculated by the hole-pipe model smaller than the leakage rate calculated by the small hole model; but the difference is not significant. Therefore, when the aperture is small, the leakage rates of the two models are very close. When the aperture increases, the effect of the decrease in the pressure at point 2 on the leakage rate will also increase. Thus, the leakage rate will not continue to increase but will tend towards being smooth and steady and will eventually be equal to the predictions of the pipeline model.

In the case of a real leak, once a leak occurs at a certain point, the flow rate (flow velocity) upstream of the leak point must increase. According to Bernoulli's equation, the flow pressure will decrease as the flow velocity increases. This effect is not considered in the hole-pipe model; thus, the leakage rate calculated by the hole-pipe model is larger than the actual leakage rate. When $d / D$ is small, the leakage rate is small, the change in flow rate is small, and the pressure of point 2 experiences minimal change compared to the healthy state. Therefore, the feedback effect typically brought on by leakage to the pressure in the pipeline upstream of the

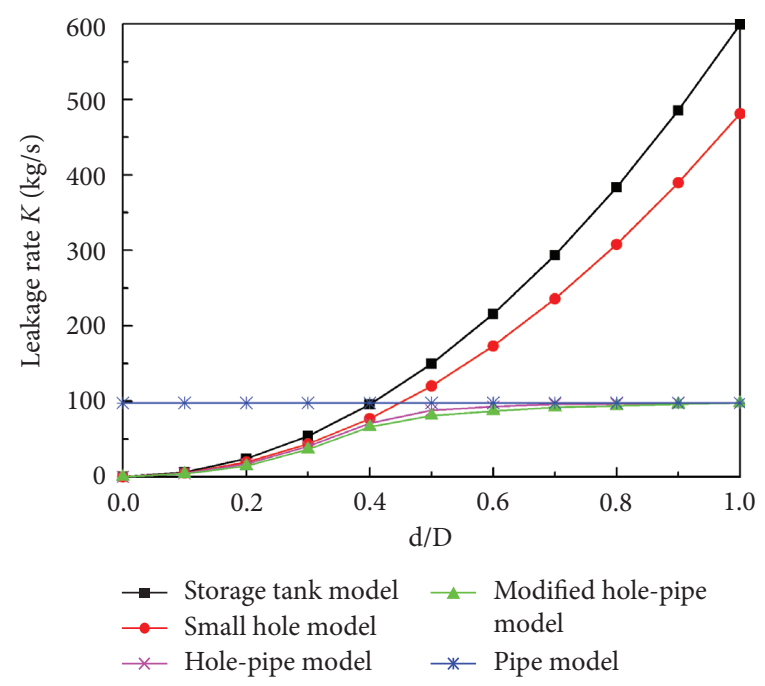

FIGURE 3: Relationship between leakage rate $K$ and d/D under different models.

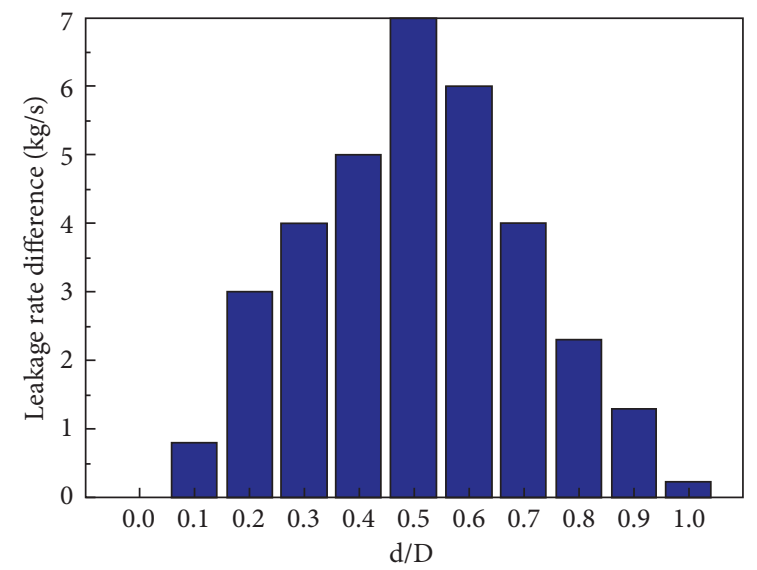

Figure 4: Leakage rate difference between the modified hole-pipe model and hole-pipe model.

leakage point can be neglected. However, as the aperture gradually increases, this feedback cannot be neglected. In the modified hole-pipe model, considering the occurrence of leakage at point 2 (as shown in Figure 1), the flow velocity in the pipeline upstream of point 2 will increase while the upstream pressure decreases. At this time, the iterative model in Figure 2 is used to calculate the new pressure and leakage rate of point 2 . The iterative model is looped until the relevant parameters of natural gas in the pipeline downstream of leakage are close to the actual situation. Thus, the leakage rate calculated by these parameters is closer to the actual leakage rate, which explains why the leakage rate calculated by the modified hole-pipe model is smaller than the leakage rate calculated by the hole-pipe model (Figure 3). With increasing $d / D$, the aperture size increases, and then, the leakage rate increases. Furthermore, the feedback effect of leakage on gas parameters (flow rate, velocity, and pressure) in pipelines becomes increasingly obvious, which makes the leakage rate difference between the modified pipehole model and the pipe-hole model increasingly larger. 
When $d / D$ approaches 1 , the aperture size is close to the diameter of the natural gas pipeline, and the pressure $P_{2}$ of point 2 is decreased and close to $P_{a}$. Subsequently, the leakage rate formula turns to equation (4), so the leakage rates calculated by these two models finally converge to a stable value together, further reducing the difference. This explains why the difference first increases and then decreases in Figure 4.

The natural gas leakage rate calculated by the pipe model is a straight line and does not vary with a change in the leakage aperture. Only when the leakage diameter is close to or equal to the diameter of the natural gas pipeline can the pipe model be applied. When the hole diameter is small, the gas leakage rate of the modified hole-pipe model is very close to the leakage rate calculated by the storage tank model or the small hole model. As d/D increases, the natural gas leakage rate increases quickly and then slowly flattens. When the leakage aperture is close to the diameter of the natural gas pipeline, the leakage rate calculated by the modified holepipe model coincides with the leakage rate calculated by the pipe model. This result indicates that the modified hole-pipe model is suitable for any leakage aperture.

Figure 5 shows the relationship between the natural gas leakage rate $K$ and $L_{e}$ (distance between point 1 and the leakage point) of the modified hole-pipe model $\left(P_{1}=18 \mathrm{MPa}\right)$ for different $\mathrm{d} / \mathrm{D}$. As the $L_{e}$ increases, the natural gas leakage rate exhibits a nonlinear downward trend. This downward trend is due to the reduction of pressure caused by friction inside the pipeline. Moreover, different leakage $d / D$ ratios have different severities. Specifically, when $d / D$ is small, the influence of the leakage on the parameters of natural gas in the pipeline is small, and the decrease in the leakage rate is smooth. When the leakage $d / D$ decreases below a certain extent, the influence of the leakage on the parameters of natural gas in the pipeline can be neglected, and the decrease in the natural gas leakage rate becomes insignificant. When the leakage $\mathrm{d} / \mathrm{D}$ increases, the curve becomes flatter. Figure 6 shows the relationship between the $K$ and $\mathrm{d} / \mathrm{D}$ as predicted by the modified hole-pipe model $\left(P_{1}=18 \mathrm{MPa}\right)$ under different $L_{e}$. It can be seen from this figure that leakage rate curves under each $L_{e}$ are similar to the leakage rate curve predicted by the modified hole-pipe model in Figure 3. Figure 6 also shows the leakage rate of the pipe model for each $L_{e}$. The figure further verifies the correctness of the modified hole-pipe model. At the same time, it can be seen from the figure that the leakage rate decreases with $L_{e}$. When $\mathrm{d} / \mathrm{D}$ is small, the leakage rate decreases slowly, and as the $d / D$ increases, the leakage rate decreases more rapidly. This is consistent with the results shown in Figure 5.

Figure 7 shows the relationship between $K$ and $P_{1}$ (the pressure of point 1) of the modified hole-pipe model $\left(L_{e}=1300 \mathrm{~m}\right)$ under different $\mathrm{d} / \mathrm{D}$. The natural gas leakage rate increased linearly with increasing $P_{1}$. Additionally, different leakage $\mathrm{d} / \mathrm{D}$ ratios lead to different levels of severities. Small $\mathrm{d} / \mathrm{D}$ leads to minimized increases in leakage rate from increases in pressure. Conversely, larger $\mathrm{d} / \mathrm{D}$ leads to higher sensitivity between pressure and leakage rate. This is because when $d / D$ is small, the influence of the leakage on

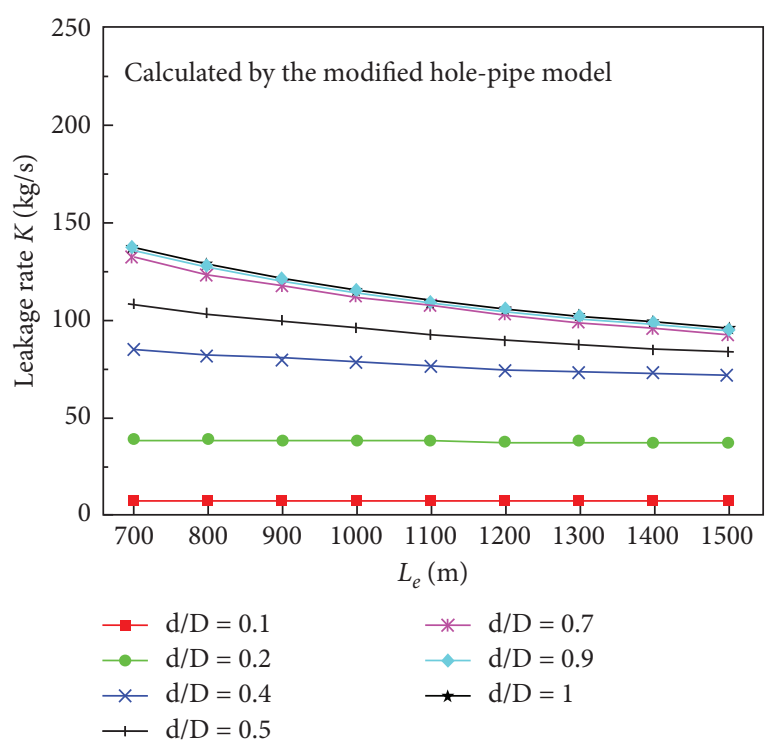

FIgURE 5: Relationship between leakage rate $K$ and $L_{e}$ under different $\mathrm{d} / \mathrm{D}\left(P_{1}=18 \mathrm{MPa}\right)$.

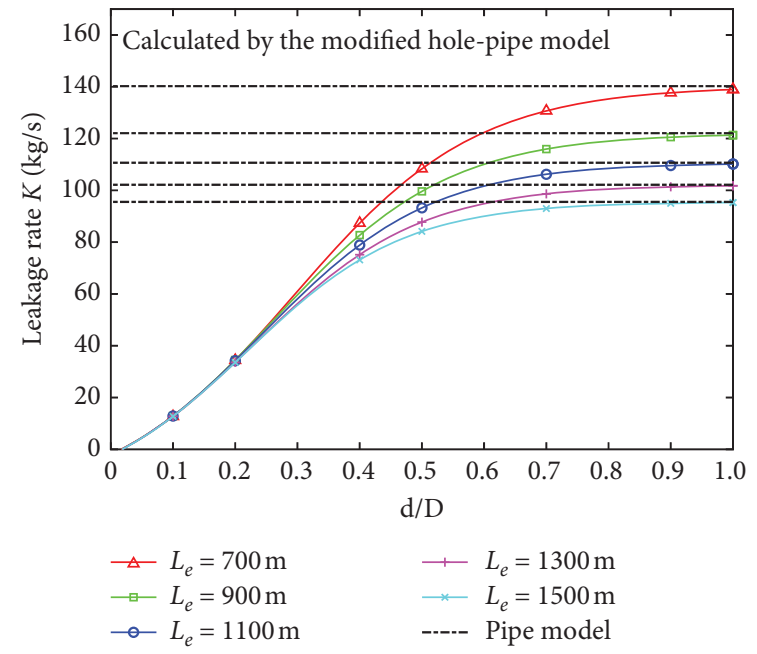

FIgURE 6: Relationship between leakage rate $K$ and d/D under different $L_{e}\left(P_{1}=18 \mathrm{MPa}\right)$.

the parameters of natural gas in the pipeline is small; when the $d / D$ is large, this influence is large. Figure 8 shows the relationship between the natural gas leakage rate $K$ and d/D of the modified hole-pipe model $\left(L_{e}=1300 \mathrm{~m}\right)$ under different $P_{1}$. It can be seen from this figure that the leakage rate curves under each $P_{1}$ are similar to the leakage rate curve predicted by the modified hole-pipe model in Figure 3. Figure 8 also shows the leakage rate of the pipe model under each $P_{1}$. The leakage rate decreases as $P_{1}$ decreases. When the $d / D$ is small, the leakage rate decreases less obviously, and as the $d / D$ increases, the leakage rate decreases more obviously. This is consistent with the results of Figure 7. The above further verifies the modified hole-pipe model.

Figures 5-8 further demonstrate the correspondence between the parameters of equation (2) and equations 


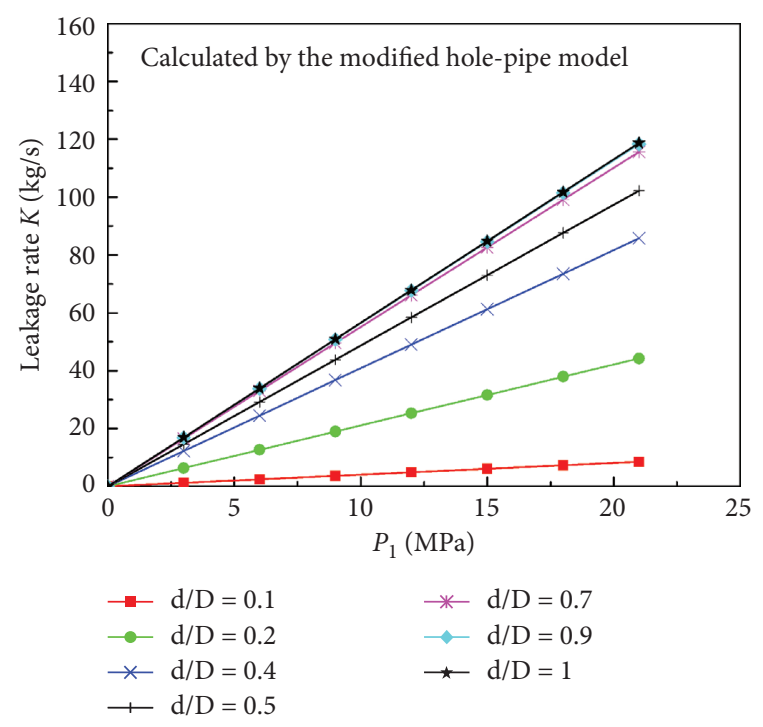

Figure 7: Relationship between leakage rate $K$ and $P_{1}$ under different $\mathrm{d} / \mathrm{D}\left(L_{e}=1300 \mathrm{~m}\right)$.

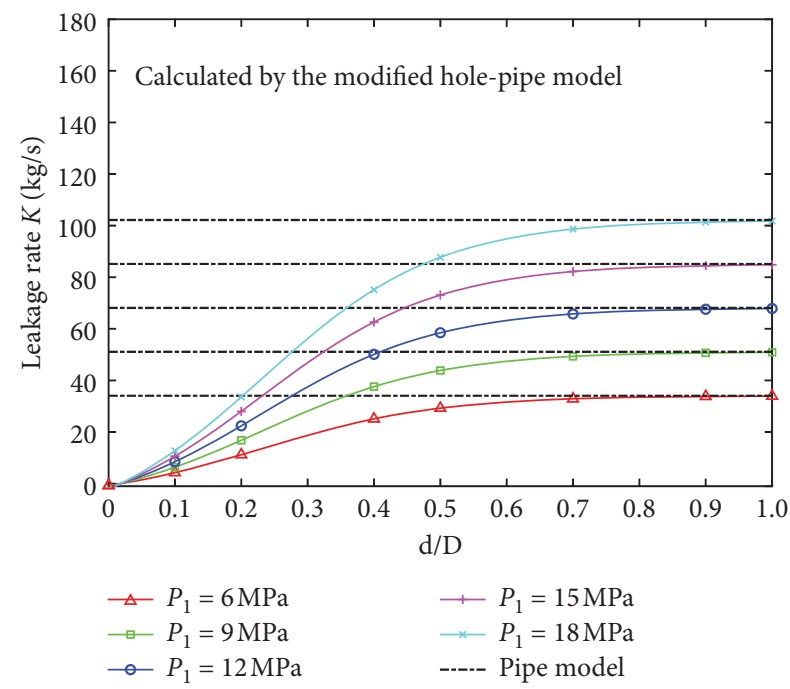

Figure 8: Relationship between leakage rate $K$ and d/D under different $P_{1}\left(L_{e}=1300 \mathrm{~m}\right)$.

(4)-(10). The relationship between $P_{2}$ ' and $d$ (diameter of the leak point hole) is shown in Figure 9.

According to the algorithm of the modified hole-pipe model shown in Section 2.4, as $d$ increases, the leakage rate $K$ increases, and therefore, the flow rate (flow velocity) upstream of the leakage point must increase because of the mass conservation. According to Bernoulli's equation, the flow pressure decreases as the flow velocity increases. From what has been discussed above, $P_{2}{ }^{\prime}$ decreases as $d$ increases, which is consistent with the trend shown in Figure 9. Furthermore, according to equations (12)-(14), before $P_{2}$ ' drops to $P_{2 c}$, the flow at the leakage point is in a state of critical flow. Once $P_{2}$ ' drops below $P_{2 c}$, the flow at the leakage point becomes subcritical flow. The demarcation point between critical flow and subcritical flow is about $d=195 \mathrm{~mm}$.

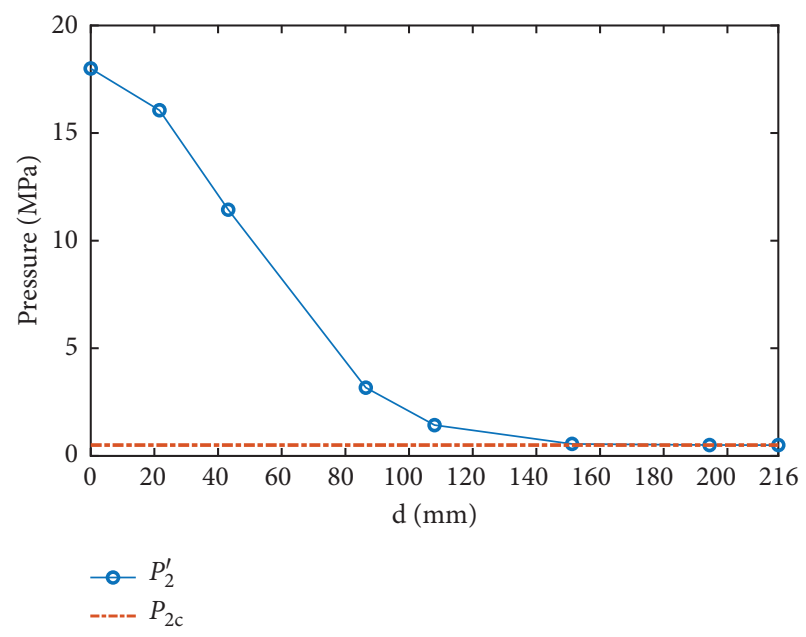

FIgURE 9: Relationship between $P_{2}$ ' and $d\left(P_{1}=18 \mathrm{MPa}\right.$ and $L_{e}=1300 \mathrm{~m}$ ).

\section{Conclusions}

This paper reclassifies the natural gas leakage models into four kinds. (a) Storage tank model-in this model, the parameters of the natural gas are considered the same as the parameters of the start point and there is no change when the leak occurs. Therefore, this model is applicable to the case where the leak point is close to the beginning of pipeline, and the leak point diameter is small; (b) small hole model-unlike the storage tank model, this model takes into account the influence of friction along the natural gas pipeline, but the pressure $P_{2}$ at point 2 is assumed to remain constant after the leakage. Therefore, this model is applicable to the case where the leak point diameter is small; (c) pipe model-this model is used for the case of the complete rupture of the natural gas pipeline or when the leak point diameter is close to the pipeline diameter; and (d) modified hole-pipe model-this model is a general model that can calculate the leakage rate at any leak point diameter.

The above classifications are more representative of the actual situation. Once the size of the leakage point can be determined, the appropriate model can be selected to estimate the leakage rate. However, the goal of most natural gas pipeline leakage problems is to determine the leakage rate as early as possible, and the leak point diameter cannot be known ahead of time. Therefore, the general model is used to estimate the leakage rate most of the time. In this case, the accuracy of the general model to calculate the leakage rate is crucial for risk assessment and failure consequence analysis. In this paper, a modified hole-pipe model is proposed, and the innovations of this modified model are as follows: (1) considering the influence of leakage on upstream flow rate change, flow rate change will produce flow velocity change. According to Bernoulli's equation, flow velocity change will produce pressure change, and pressure change will further affect the leakage rate, which is a loop problem. This influence is not taken into account in the hole-pipe model, but it is 
taken into account in this modified model by using the loop iterative algorithm of successive approximation. (2) In the hole-pipe model, the influence of leakage on the leak point pressure has been considered, but complex hydrodynamics and thermodynamics calculations are required. In this modified hole-pipe model, the successive approximation iterative loop algorithm can take the influence of leakage on the leak point pressure into account and avoids complex computation due to the advantages of the algorithm. A comparison and analysis of the calculation results in this paper shows that the modified hole-pipe model, like the hole-pipe model, is a general model that can calculate the leakage rate at any leak point diameter, and the leakage rate calculated by the modified hole-pipe model is lower than that calculated by the hole-pipe model, which conforms to the theoretical analysis and should be closer to the actual leakage rate. Therefore, the modified hole-pipe model proposed in this paper can calculate the leakage rate more accurately.

\section{Nomenclature}

$P: \quad$ Pressure $(\mathrm{Pa})$

$P_{i}: \quad$ Pressure for each point $(\mathrm{Pa})$

$P_{a}: \quad$ Pressure for surrounding $(\mathrm{Pa})$

$P_{2 c}$ : Critical pressure at point $2(\mathrm{~Pa})$

$T: \quad$ Temperature $(K)$

$T_{i}$ : $\quad$ Temperature for each point $(K)$

$u_{i}$ : Flow velocity $(\mathrm{m} / \mathrm{s})$

$G: \quad$ Mass flux $\left(\mathrm{kg} / \mathrm{m}^{2} \cdot \mathrm{s}\right)$

$k$ : Heat capacity ratio

$Q: \quad$ Flow rate before leakage point $(\mathrm{kg} / \mathrm{s})$

$\mathrm{Q}^{+}$: $\quad$ Flow rate after leakage point $(\mathrm{kg} / \mathrm{s})$

$K$ : Leakage rate at the leakage point $(\mathrm{kg} / \mathrm{s})$

$M: \quad$ Molar mass $(\mathrm{kg} / \mathrm{mol})$

$R: \quad$ Ideal gas constant $(\mathrm{J} /(\mathrm{mol} \cdot \mathrm{K}))$

$X_{e}: \quad$ Equivalent between point 1 and point $2(\mathrm{~m})$

$D: \quad$ Inner diameter of pipes $(\mathrm{m})$

$\rho: \quad$ Density of gas $\left(\mathrm{kg} / \mathrm{m}^{3}\right)$

$E$ : Roughness of pipes $(\mathrm{mm})$

Re: Reynolds number

$\lambda$ : $\quad$ Fanning friction factor

$A_{\text {or: }}$ : Hole area $\left(\mathrm{m}^{2}\right)$

$C_{D}$ : Hole flow correction coefficient

$Z$ : $\quad$ Air compression factor

CPR: Critical pressure ratio

$L_{e}$ : Distance between point 1 and point $2(\mathrm{~m})$

$D$ : Diameter of the pipeline $(\mathrm{mm})$

$D$ : Diameter of the leak point hole $(\mathrm{mm})$.

\section{Data Availability}

The data used to support the findings of this study are available from the corresponding author upon request.

\section{Conflicts of Interest}

The authors declare that they have no conflicts of interest.

\section{Acknowledgments}

This research was funded by Scientific Research Project of Harbin University of Commerce (No. 17XN013) entitled "Research about detection and location of natural gas pipeline leakages based on Kalman filter and Fiber sensing" and the Doctoral Research Project of Harbin University of Commerce (No. 2016BS19).

\section{References}

[1] H. R. Zhang, Y. T. Liang, W. Zhang, N. Xu, Z. L. Guo, and G. M. Wu, "Improved pso-based method for leak detection and localization in liquid pipelines," IEEE Transactions on Industrial Informatics, vol. 14, pp. 3143-3154, 2018.

[2] H. R. Zhang, Y. T. Liang, Q. Liao, S. Yun, and X. H. Yang, “A self-learning approach for optimal detailed scheduling of multi-product pipeline," Journal of Computational and Applied Mathematics, vol. 327, pp. 41-63, 2018.

[3] Q. Liao, H. Zhang, N. Xu, Y. Liang, and J. Wang, "A milp model based on flowrate database for detailed scheduling of a multi-product pipeline with multiple pump stations," Computers \& Chemical Engineering, vol. 117, pp. 63-81, 2018.

[4] Q. Liao, P. M. Castro, Y. T. Liang, and H. R. Zhang, "New batch-centric model for detailed scheduling and inventory management of mesh pipeline networks," Computers \& Chemical. Engineering, vol. 130, 2019.

[5] Z. B. Li, H. X. Feng, Y. T. Liang, N. Xu, S. M. Nie, and H. R. Zhang, "A leakage risk assessment method for hazardous liquid pipeline based on markov chain monte carlo," International Journal of Critical Infrastructure Protection, vol. 27, 2019.

[6] B. Wang, Y. Liang, J. Zheng, T. Lei, M. Yuan, and H. Zhang, "A methodology to restructure a pipeline system for an oilfield in the mid to late stages of development," Computers \& Chemical Engineering, vol. 115, pp. 133-140, 2018.

[7] A. Chebouba, F. Yalaoui, A. Smati, L. Amodeo, K. Younsi, and A. Tairi, "Optimization of natural gas pipeline transportation using ant colony optimization," Computers \& Operations Research, vol. 36, no. 6, pp. 1916-1923, 2009.

[8] Q. Ji, Q. Kong, and G. Song, "Study on energy focusing synthesis on pipe using time reversal technique," in Proceedings of the 11th IEEE International Conference on Networking, Sensing and Control, pp. 625-630, Miami, FL, USA, 2014.

[9] G. Park, H. H. Cudney, D. J. Inman, and J. I. Daniel, "Feasibility of using impedance-based damage assessment for pipeline structures," Earthquake Engineering \& Structural Dynamics, vol. 30, no. 10, pp. 1463-1474, 2001.

[10] Q. Ji, S. M. Parvasi, S. C. M. Ho, M. Franchek, and G. Song, "Wireless energy harvesting using time reversal technique: an experimental study with numerical verification," Journal of Intelligent Material Systems and Structures, vol. 28, no. 19, pp. 2705-2716, 2017.

[11] H. Montiel, J. Vilchez, J. Casal, and J. Arnaldos, "Mathematical modelling of accidental gas releases," Journal of Hazardous Materials, vol. 59, no. 2, pp. 211-233, 1998.

[12] Z. Zhou, "Thermal analysis of slow discharge from a pressurized natural gas storage tank," Applied Thermal Engineering, vol. 17, no. 11, pp. 1099-1110, 1997.

[13] Z. Zhou, B. Smith, and G. Yadigaroglu, "A mathematical model and its analytical solution for slow depressurization of a gas-filled vessel," Journal of Engineering Mathematics, vol. 31, no. 1, pp. 43-57, 1997. 
[14] J. L. Woodward and K. S. Mudan, "Liquid and gas discharge rates through holes in process vessels," Journal of Loss Prevention in the Process Industries, vol. 4, no. 4, pp. 161-165, 1991.

[15] CCPS, Guidelines for Use of Vapor Cloud Dispersion Models, AICE, New York, NY, USA, 2 edition, 1996.

[16] J. D. Young and A. J. Bum, "A simple model for the release rate of hazardous gas from a hole on high-pressure pilelines," Journal of Hazardous Materials, vol. 97, pp. 31-46, 2003.

[17] J. D. Young and A. J. Bum, "Analysis of hazard areas associated with high-pressure natural-gas pipelines," Journal of Prevention in the Process Industries, vol. 15, no. 3, pp. 179-198, 2002.

[18] J. Arnaldos, J. Casal, H. Montiel, M. Sánchez-Carricondo, and J. A. V'llchez, "Design of a computer tool for the evaluation of the consequences of accidental natural gas releases in distribution pipes," Journal of Loss Prevention in the Process Industries, vol. 11, no. 2, pp. 135-148, 1998.

[19] O. Levenspiel, Engineering Flow and Heat Exchange, Plenum, New York, NY, USA, 1984.

[20] Y. H. Dong, "The calculation of the steady-state rate of gas leakage in long-distance pipeline," Oil and Gas Storage and Transportation, vol. 34, pp. 11-15, 2002.

[21] Z. Yang, F. R. Zhang, and J. B. Lai, "Steady leakage calculation models of non-isothermal long gas pipeline," Journal of Tianjin University of Technology, vol. 38, pp. 1115-1121, 2005.

[22] K. Wojciech J and S. Janusz, "Real gas flow simulation in damaged distribution pipelines," Energy, vol. 45, pp. 481-488, 2012.

[23] N. Bariha, I. M. Mishra, and V. C. Srivastava, "Hazard analysis of failure of natural gas and petroleum gas pipelines," Journal of Loss Prevention in the Process Industries, vol. 40, pp. 217226, 2016. 